KYUNGPOOK Math. J. 55(2015), 449-472

http://dx.doi.org/10.5666/KMJ.2015.55.2.449

pISSN 1225-6951 eISSN 0454-8124

(C) Kyungpook Mathematical Journal

\title{
Extended by Balk Metrics
}

\section{Oleksiy Dovgoshey}

Division of Applied Problems in Contemporary Analysis, Institute Mathematics of NASU, Tereschenkivska str. 3, Kiev - 4, 01601, Ukraine

Donetsk National University, 600-letiya Street 21, Vinnitsa, Ukraine

e-mail : aleksdov@mail.ru

DMYTRO DORDOVSKYI*

Institute of Applied Mathematics and Mechanics of NASU, R. Luxemburg str. 74, Donetsk, 83114, Ukraine

e-mail : dordovskydmitry@gmail.com

Abstract. Let $X$ be a nonempty set and $\mathcal{F}(X)$ be the set of nonempty finite subsets of $X$. The paper deals with the extended metrics $\tau: \mathcal{F}(X) \rightarrow \mathbb{R}$ recently introduced by Peter Balk. Balk's metrics and their restriction to the family of sets $A$ with $|A| \leqslant n$ make possible to consider "distance functions" with $n$ variables and related them quantities. In particular, we study such type generalized diameters $\operatorname{diam}_{\tau^{n}}$ and find conditions under which $B \mapsto \operatorname{diam}_{\tau^{n}} B$ is a Balk's metric. We prove the necessary and sufficient conditions under which the restriction $\tau$ to the set of $A \in \mathcal{F}(X)$ with $|A| \leqslant 3$ is a symmetric $G$-metric. An infinitesimal analog for extended by Balk metrics is constructed.

\section{Introduction}

The following generalized metrics were introduced by P. Balk in 2009 for applications to some inverse geophysical problems $([2])$. $X$.

Let $X$ be a nonempty set and $\mathcal{F}(X)$ be the set of all nonempty finite subsets of

Definition 1.1.([3]) A function $\tau: \mathcal{F}(X) \rightarrow \mathbb{R}$ is an extended (by Balk) metric on $X$ if the equivalence

$$
(\tau(A)=0) \Leftrightarrow(|A|=1),
$$

* Corresponding Author.

Received December 21, 2013; accepted April 11, 2014.

2010 Mathematics Subject Classification: 54E35.

Key words and phrases: generalized diameter, $G$-metric, pretangent space, ultrametric, ultrafilter. 
and the equality

$$
\tau(A \cup B) \leqslant \tau(A \cup C)+\tau(C \cup B)
$$

hold for all $A, B, C \in \mathcal{F}(X)$.

Example 1.2.([3]) If $\rho$ is a metric on $X$, then the function $\tau(A)=\operatorname{diam}_{\rho}(A)$, with $\operatorname{diam}_{\rho}(A)=\sup \{\rho(x, y): x, y \in A\}$, is an extended by Balk metric.

If $\tau$ is an extended by Balk metric on $X$ then, as shown in Proposition 2.1, the function $\tau^{2}: X^{2} \rightarrow \mathbb{R}$, with

$$
\tau^{2}(x, y)=\tau(\operatorname{Im}(x, y)), \quad \operatorname{Im}(x, y)= \begin{cases}\{x\} & \text { if } x=y \\ \{x, y\} & \text { if } x \neq y\end{cases}
$$

is a metric on $X$. Analogously, for all integer numbers $k \geqslant 1$ we can define the functions $\tau^{k}: X^{k} \rightarrow \mathbb{R}$ as

$$
\tau^{k}\left(x_{1}, \ldots, x_{k}\right)=\tau\left(\operatorname{Im}\left(x_{1}, \ldots, x_{k}\right)\right),
$$

where $\operatorname{Im}\left(x_{1}, \ldots, x_{k}\right)$ is the image of the set $\{1, \ldots, k\}$ under the map $i \mapsto x_{i}$,

$$
\left(x \in \operatorname{Im}\left(x_{1}, \ldots, x_{k}\right)\right) \Leftrightarrow\left(\exists i \in\{1, \ldots, k\}: x=x_{i}\right) .
$$

Formula (1.4) turns to formula (1.3) when $k=2$, thus we obtain a "generalized metric" which is a function of $k$ variables (while the usual metric is a function of two variables).

In what follows the important role will play some "generalized diameters" generated by $\tau^{k}$.

Definition 1.3. Let $X \neq \varnothing, k$ be an integer positive number and let $\tau: \mathcal{F}(X) \rightarrow \mathbb{R}$ be an extended by Balk metric. For every nonempty $A \subseteq X$ we set

$$
\operatorname{diam}_{\tau^{k}} A=\sup \left\{\tau^{k}\left(x_{1}, \ldots, x_{k}\right): x_{1}, \ldots, x_{k} \in A\right\}
$$

that is equivalent to

$$
\operatorname{diam}_{\tau^{k}} A=\sup \{\tau(B): B \subseteq A,|B| \leqslant k\} .
$$

Remark 1.4. It is clear that $\operatorname{diam}_{\tau^{k}} A$ is the usual diameter of $A$ if $k=2$. Definition 1.1 implies $\operatorname{diam}_{\tau^{1}} A=0$ for every $A \in \mathcal{F}(X)$.

In Theorem 2.12 of the second section of the paper we obtain a structural characteristic of extended by Balk metrics $\tau: \mathcal{F}(X) \rightarrow \mathbb{R}$ for which $\tau(A)=\operatorname{diam}_{\tau^{k}} A$ holds with all $A \in \mathcal{F}(X)$ and $k \geqslant 2$.

In the third section we study the relationship between $\tau^{3}$ and the so-called $G$-metrics which were introduced by Zead Mustafa and Brailey Sims in 2006.

Definition 1.5.([20]) Let $X$ be a nonempty set. A function $G: X^{3} \rightarrow \mathbb{R}$ is called a $G$-metric if the following properties hold. 
(i) $G(x, y, z)=0$ for $x=y=z$.

(ii) $0<G(x, x, y)$ for $x \neq y$.

(iii) $G(x, x, y) \leqslant G(x, y, z)$ for $z \neq y$.

(iv) $G\left(x_{1}, x_{2}, x_{3}\right)=G\left(x_{\sigma_{1}}, x_{\sigma_{2}}, x_{\sigma_{3}}\right)$ for every permutation $\sigma$ of the set $\{1,2,3\}$ and every $\left(x_{1}, x_{2}, x_{3}\right) \in X^{3}$.

(v) $G(x, y, z) \leqslant G(x, a, a)+G(a, y, z)$ for all $a, x, y, z \in X$.

Definition 1.6. A $G$-metric is called symmetric if the equality $G(x, y, y)=$ $G(y, x, x)$ holds for all $x, y \in X$.

Remark 1.7. In [20] $G$-metrics were defined as some functions $G$ with the codomain $[0, \infty)$, which is slightly different from Definition 1.5 . In this connection it should be pointed out that conditions $(i)-(i v)$ of Definition 1.5 imply the nonnegativity of $G$. Indeed, it is sufficient to prove $G(y, x, x)>0$ for $x \neq y$, that follows from $0<G(x, x, y)=G(x, y, x)=G(y, x, x)$.

We shall prove that for every symmetric $G$-metric on $X$ there is an increasing extended by Balk metric $\tau: \mathcal{F}(X) \rightarrow \mathbb{R}$ such that $\tau^{3}=G$. Conversely, an arbitrary $\tau^{3}$ is a $G$-metric if the corresponding extended by Balk metric $\tau: \mathcal{F}(X) \rightarrow \mathbb{R}$ is increasing. (See Theorem 3.7).

The infinitesimal structure of spaces $(X, \tau)$ with extended by Balk metrics $\tau$ is investigated in the fourth section. In particular, we transfer the extended by Balk metrics $\tau$ from $X$ to spaces which are pretangent to $\left(X, \tau^{2}\right)$. The pretangent spaces to the general metric spaces were introduced in [12] (see also [13]). For convenience, we recall some related definitions.

Let $(X, d)$ be a metric space and let $p \in X$. Fix a sequence $\tilde{r}$ of positive real numbers $r_{n}$ which tend to zero. The sequence $\tilde{r}$ will be called a normalizing sequence. Let us denote by $\tilde{X}_{p}$ the set of all sequences of points from $X$ which tend to $p$.

Definition 1.8. Two sequences $\tilde{x}, \tilde{y} \in \tilde{X}_{p}, \tilde{x}=\left(x_{n}\right)_{n \in \mathbb{N}}$ and $\tilde{y}=\left(y_{n}\right)_{n \in \mathbb{N}}$ are mutually stable with respect to a normalizing sequence $\tilde{r}=\left(r_{n}\right)_{n \in \mathbb{N}}$, if there is a finite limit

$$
\lim _{n \rightarrow \infty} \frac{d\left(x_{n}, y_{n}\right)}{r_{n}}:=\tilde{d}_{\tilde{r}}(\tilde{x}, \tilde{y})=\tilde{d}(\tilde{x}, \tilde{y})
$$

The family $\tilde{F} \subseteq \tilde{X}_{p}$ is self-stable with respect to $\tilde{r}$, if every two $\tilde{x}, \tilde{y} \in \tilde{F}$ are mutually stable, $\tilde{F}$ is maximal self-stable if $\tilde{F}$ is self-stable and for an arbitrary $\tilde{z} \in \tilde{X}_{p} \backslash \tilde{F}$ there is $\tilde{x} \in \tilde{F}$ such that $\tilde{x}$ and $\tilde{z}$ are not mutually stable. Zorn's lemma leads to the following

Proposition 1.9. Let $(X, d)$ be a metric space and let $p \in X$. Then for every normalizing sequence $\tilde{r}=\left\{r_{n}\right\}_{n \in \mathbb{N}}$ there exists a maximal self-stable family $\tilde{X}_{p, \tilde{r}}$ such that $\tilde{p}=\{p, p, \ldots\} \in \tilde{X}_{p, \tilde{r}}$. 
Let us consider a function $\tilde{d}: \tilde{X}_{p, \tilde{r}} \times \tilde{X}_{p, \tilde{r}} \rightarrow \mathbb{R}$, where $\tilde{d}(\tilde{x}, \tilde{y})=\tilde{d}_{\tilde{r}}(\tilde{x}, \tilde{y})$ is defined by (1.7). Obviously, $\tilde{d}$ is symmetric and nonnegative. Moreover, the triangle inequality for $d$ implies

$$
\tilde{d}(\tilde{x}, \tilde{y}) \leq \tilde{d}(\tilde{x}, \tilde{z})+\tilde{d}(\tilde{z}, \tilde{y})
$$

for all $\tilde{x}, \tilde{y}, \tilde{z} \in \tilde{X}_{p, \tilde{r}}$. Hence $\left(\tilde{X}_{p, \tilde{r}}, \tilde{d}\right)$ is a pseudometric space.

Define a relation $\sim$ on $\tilde{X}_{p, \tilde{r}}$ by $\tilde{x} \sim \tilde{y}$ if and only if $\tilde{d}_{\tilde{r}}(\tilde{x}, \tilde{y})=0$. Let us denote by $\Omega_{p, \tilde{r}}^{X}$ the set of equivalence classes in $\tilde{X}_{p, \tilde{r}}$ under the equivalence relation $\sim$. For $\alpha, \beta \in \Omega_{p, \tilde{r}}^{X}$ set

$$
\rho(\alpha, \beta)=\tilde{d}(\tilde{x}, \tilde{y})
$$

where $\tilde{x} \in \alpha$ and $\tilde{y} \in \beta$, then $\rho$ is a metric on $\Omega_{p, \tilde{r}}^{X}$ (see, for example, [16, Ch. 4 , Theorem 15]).

Definition 1.10. The space $\left(\Omega_{p, \tilde{r}}^{X}, \rho\right)$ is pretangent to the space $X$ at the point $p$ with respect to a normalizing sequence $\tilde{r}$.

Let $\tau: \mathcal{F}(X) \rightarrow \mathbb{R}$ be an extended by Balk metric, let $p \in X$ and let $\left(\Omega_{p, \tilde{r}}^{X}, \rho\right)$ be a pretangent space to the metric space $\left(X, \tau^{2}\right)$. Now the "lifting" of $\tau$ on $\left(\Omega_{p, \tilde{r}}^{X}, \rho\right)$ is defined as follows. Let $\mathfrak{U}$ be a nontrivial ultrafilter on $\mathbb{N}$. For $\left\{\alpha_{1}, \ldots, \alpha_{n}\right\} \in \mathcal{F}\left(\Omega_{p, \tilde{r}}^{X}\right)$, $\left(x_{m}^{1}\right)_{m \in \mathbb{N}} \in \alpha_{1}, \ldots,\left(x_{m}^{n}\right)_{m \in \mathbb{N}} \in \alpha_{n}$ set

$$
x_{\tau}\left(\left\{\alpha_{1}, \ldots, \alpha_{n}\right\}\right)=\mathfrak{U}-\lim \frac{\tau\left(\operatorname{Im}\left(x_{m}^{1}, \ldots, x_{m}^{n}\right)\right)}{r_{m}} .
$$

In Theorem 4.3 it is proved that $X_{\tau}$ is an extended by Balk metric on $\left(\Omega_{p, \tilde{r}}^{X}, \rho\right)$ and $X_{\tau}^{2}=\rho$. Theorem 4.8 provides a characteristic of extended metrics $\tau: \mathcal{F}(X) \rightarrow \mathbb{R}$ for which the equality

$$
x_{\tau}(A)=\operatorname{diam}_{\rho} A
$$

holds for every $A \in \mathcal{F}\left(\Omega_{p, \tilde{r}}^{X}\right)$. This result is used in Corollary 4.10 for characterization of $\tau$ for which $X_{\tau}$ are the extended "ultrametrics", i.e. satisfy the inequality

$$
x_{\tau}(A \cup B) \leqslant \max \left\{x_{\tau}(A \cup C), \chi_{\tau}(B \cup C)\right\}
$$

instead of inequality (1.2).

\section{Extended by Balk Metrics and Generalized Diameters}

Let $X$ be a nonempty set and $\tau: \mathcal{F}(X) \rightarrow \mathbb{R}$ be an extended by Balk metric on $X$. Set

$$
\tau^{2}(x, y):= \begin{cases}\tau(\{x, y\}), & \text { if } x \neq y \\ 0, & \text { if } \mathrm{x}=\mathrm{y}\end{cases}
$$


for every ordered pair $(x, y) \in X \times X$, where $\{x, y\}$ is the set whose elements are the points $x$ and $y$.

Proposition 2.1. The function $\tau^{2}: X^{2} \rightarrow \mathbb{R}$ is a metric for every nonempty set $X$ and extended metric $\tau: \mathcal{F}(X) \rightarrow \mathbb{R}$.

Proof. Obviously, the function $\tau^{2}$ is symmetric and by $(1.1) \tau^{2}(x, y)=0$ if and only if $x=y$. Putting in (1.2) $A=B=C$ we obtain $\tau(A) \leqslant 2 \tau(A)$ for every $A \in \mathcal{F}(X)$ that is an equivalent to $\tau(A) \geqslant 0$. The last inequality implies the nonnegativity of the function $\tau^{2}$. It remains to prove the triangle inequality for $\tau^{2}$. Let $x, y, z$ be arbitrary points from $X$. Putting $A=\{x\}, B=\{y\}$ and $C=\{z\}$ into inequality (1.2) we obtain

$$
\begin{gathered}
\tau^{2}(x, y)=\tau(\{x\} \cup\{y\}) \leqslant \tau(\{x\} \cup\{z\})+\tau(\{z\} \cup\{y\}) \\
\leqslant \tau(\{x, z\})+\tau(\{z, y\})=\tau^{2}(x, z)+\tau^{2}(z, y) .
\end{gathered}
$$

Thus the triangle inequality is satisfied.

If $d$ is a metric and $\tau$ is an extended by Balk metric on the same set $X$ and the equality $d(x, y)=\tau^{2}(x, y)$ holds for all $x, y \in X$, we say that $\tau$ is compatible with $d$.

Remark 2.2. The nonnegativity of $\tau$ was earlier proved in [3].

Recall that a mapping $f: X \rightarrow Y$ from a partially ordered set $\left(X, \leqslant_{X}\right)$ to a partially ordered set $\left(Y, \leqslant_{Y}\right)$ is called increasing if the implication

$$
\left(x \leqslant_{X} y\right) \Rightarrow\left(f(x) \leqslant_{Y} f(y)\right)
$$

holds for all $x, y \in X$.

Let us put in order the set $\mathcal{F}(X)$ by the set-theoretic inclusion $\subseteq$ and consider $\mathbb{R}$ with the standard order $\leqslant$. If $\rho$ is a metric on $X$, then the mapping

$$
\mathcal{F}(X) \ni A \mapsto \operatorname{diam}_{\rho}(A) \in \mathbb{R}
$$

is increasing.

Definition 2.3. Let $X \neq \varnothing$ and $k$ be an integer number greater or equal two. A mapping $f: \mathcal{F}(X) \rightarrow \mathbb{R}$ is called $k$-increasing if the implication

$$
(B \subseteq A) \Rightarrow(f(B) \leqslant f(A))
$$

holds for $A, B \in \mathcal{F}(X)$ with $|B| \leqslant k$.

Remark 2.4. It is clear that every increasing mapping $f: \mathcal{F}(X) \rightarrow \mathbb{R}$ is $k$ increasing for every $k \geqslant 2$. It is not hard to check that, if $|X| \leqslant k+1$, then all $k$-increasing mappings are increasing.

The next example shows that for $|X| \geqslant k+2$ there are extended by Balk metrics on $X$ which are $k$-increasing but not $k+1$-increasing mappings. 
Example 2.5. Let $|X| \geqslant k+2$ and $t_{i}, i=2, \ldots, k+2$ be some numbers from the interval $(1,2)$ such that $t_{k}<t_{k+2}<t_{k+1}$ and $t_{i}<t_{i+1}$ for $i=2, \ldots, k$. For $A \in \mathcal{F}(X)$ set

$$
\tau(A)= \begin{cases}0, & \text { for }|A|=1 \\ t_{n}, & \text { for }|A|=n, \text { if } 2 \leqslant n \leqslant k+1 \\ t_{k+2}, & \text { for }|A| \geqslant k+2\end{cases}
$$

It follows directly from (2.2) and the restrictions to the numbers $t_{n}$ that $\tau$ is $k$ increasing but not $k+1$-increasing. If $|A \cup C| \neq 1 \neq|B \cup C|$ and $\tau(A \cup B)=t_{i}$, $\tau(A \cup C)=t_{j}, \tau(B \cup C)=t_{l}$ then $t_{i}, t_{j}, t_{l} \in(1,2)$. Hence $t_{i} \leqslant t_{j}+t_{l}$ that implies (1.2). Assuming, for example, the equality $1=|B \cup C|$ we obtain the existence of $x \in X$ such that $B=C=\{x\}$. Then inequality (1.2) turns into an equality. Case $|A \cup C|=1$ is similar.

Lemma 2.6. The following conditions are equivalent for all $X \neq \varnothing, \tau: \mathcal{F}(X) \rightarrow \mathbb{R}$ and integer numbers $k \geqslant 2$.

(i) The mapping $\tau$ is a k-increasing function from $(\mathcal{F}(X), \subseteq)$ to $(\mathbb{R}, \leqslant)$.

(ii) The inequality

$$
\tau(A) \geqslant \max \{\tau(B): B \subseteq A,|B| \leqslant k\}
$$

holds for every $A \in \mathcal{F}(X)$.

The proof can be obtained directly from definitions and we omit it here.

Corollary 2.7. Let $X \neq \varnothing$ and $k$ be an integer number greater or equal two. An extended metric $\tau: \mathcal{F}(X) \rightarrow \mathbb{R}$ is a k-increasing mapping from $(\mathcal{F}(X), \subseteq)$ to $(\mathbb{R}, \leqslant)$ if and only if the inequality $\tau(A) \geqslant \operatorname{diam}_{\tau^{k}} A$ holds for every $A \in \mathcal{F}(X)$ where $\operatorname{diam}_{\tau^{k}} A$ is defined by relation (1.6).

Let $\left(X, \leqslant_{X}\right)$ and $\left(Y, \leqslant_{Y}\right)$ be partially ordered sets. A mapping $f: X \rightarrow Y$ is called decreasing if the implication $(z \leqslant X \quad y) \Rightarrow\left(f(z) \geqslant_{Y} f(y)\right)$ holds for all $z, y \in X$.

In the following definition the relation $B \subset A$ means that we have $B \subseteq A$ and $B \neq A$.

Definition 2.8. Let $X \neq \varnothing$ and $k \geqslant 2$ be an integer number. A mapping $f$ : $\mathcal{F}(X) \rightarrow \mathbb{R}$ is $k$-weakly decreasing if for every $A \in \mathcal{F}(X)$ with $|A|>k$ there is a finite nonempty set $B \subset A$ such that $f(B) \geqslant f(A)$.

Lemma 2.9. The following conditions are equivalent for all $X \neq \varnothing, k \geqslant 2$ and mappings $\tau: \mathcal{F}(X) \rightarrow \mathbb{R}$.

(i) The mapping $\tau$ is k-weakly decreasing. 
(ii) The inequality

$$
\tau(A) \leqslant \max \{\tau(B): B \subseteq A,|B| \leqslant k\}
$$

holds for every $A \in \mathcal{F}(X)$.

Proof. The implication (ii) $\Rightarrow$ (i) follows directly from Definition 2.8. Let us check the implication (i) $\Rightarrow$ (ii). Assume that condition (i) is true. Let us prove inequality (2.4) using induction by $|A|$. If $|A|=1, \ldots, k$ inequality (2.4) is obvious. Suppose that (2.4) is proved for $|A| \leqslant n, n \in \mathbb{N}$. Assume $|A|=n+1 \geqslant k+1$. By (i) the mapping $\tau$ is $k$-weakly decreasing. Therefore there is $B \subset A$ such that $\tau(A) \leqslant \tau(B)$. From the inclusion $B \subset A$ follows the inequality $|B| \leqslant n$. Using the induction hypothesis we get

$$
\tau(A) \leqslant \tau(B) \leqslant \max \{\tau(C): C \subseteq B,|C| \leqslant k\} .
$$

Since $(C \subseteq B)$ implies $(C \subseteq A)$, we obtain

$$
\max \{\tau(C): C \subseteq B,|C| \leqslant k\} \leqslant \max \{\tau(C): C \subseteq A,|C| \leqslant k\} .
$$

The last inequality and (2.5) give (2.4).

The next corollary directly follows from Definition 1.3 and Lemma 2.9.

Corollary 2.10. Let $X \neq \varnothing$ and $k \geqslant 2$ be an integer number. An extended by Balk metric $\tau: \mathcal{F}(X) \rightarrow \mathbb{R}$ is a k-weakly decreasing mapping from $(\mathcal{F}(X), \subseteq)$ into $(\mathbb{R}, \leqslant)$ if and only if the inequality $\tau(A) \leqslant \operatorname{diam}_{\tau^{k}}(A)$ holds for every $A \in \mathcal{F}(X)$.

Lemmas 2.6 and 2.9 give the following.

Corollary 2.11. Let $X \neq \varnothing$, let $k \geqslant 2$ be an integer number and let $\tau: \mathcal{F}(X) \rightarrow \mathbb{R}$ be a $k$-weakly decreasing mapping. Then $\tau$ is increasing if and only if it is $k$ increasing.

Proof. It is sufficient to verify that if $\tau$ is $k$-increasing then $\tau$ is increasing. Indeed, if $\tau$ is $k$-increasing, then inequalities (2.3) and (2.4) imply

$$
\tau(A)=\max \{\tau(B): B \subseteq A,|B| \leqslant k\}, \quad A \in \mathcal{F}(A) .
$$

The increase of $\tau$ follows.

Combining corollaries 2.7, 2.10 and 2.11 we get

Theorem 2.12. The following statements are equivalent for all nonempty $X$, integer $k \geqslant 2$ and extended metrics $\tau: \mathcal{F}(X) \rightarrow \mathbb{R}$.

(i) The equality $\tau(A)=\operatorname{diam}_{\tau^{k}} A$ holds for every $A \in \mathcal{F}(X)$, where $\operatorname{diam}_{\tau^{k}} A$ is determined by Definition 1.3.

(ii) $\tau$ is $k$-increasing and $k$-weakly decreasing. 
(iii) $\tau$ is increasing and $k$-weakly decreasing.

Definition 2.13. Let $\rho$ be a metric on $X$ and $\tau: \mathcal{F}(X) \rightarrow \mathbb{R}$ be an extended by Balk metric on $X$. We say that $\tau$ is generated by $\rho$ if $\tau(A)=\operatorname{diam}_{\rho} A$ for any $A \in \mathcal{F}(A)$.

Theorem 2.14. Let $\tau: \mathcal{F}(X) \rightarrow \mathbb{R}$ be an extended by Balk metric on a nonempty set $X$. The following statements are equivalent.

(i) There is a mapping $\mu: X^{2} \rightarrow \mathbb{R}$ such that $\mathcal{F}(A)=\max \{\mu(x, y): x, y \in A\}$ for every $A \in \mathcal{F}(X)$.

(ii) There is a metric on $X$ which generates $\tau$.

(iii) $\tau$ is generated by $\tau^{2}$.

(iv) $\tau$ is 2-increasing and 2-weakly decreasing.

(v) $\tau$ is increasing and 2-weakly decreasing.

Proof. The implications (iii) $\Rightarrow$ (ii) and (ii) $\Rightarrow$ (i) are obvious. The equivalences (v) $\Leftrightarrow$ (iv) and (iv) $\Leftrightarrow$ (iii) follow from Theorem 2.12. It remains to note that (i) $\Leftrightarrow$ (v) follows immediately from the definitions of increasing mapping and 2weakly decreasing one.

In the next section we will prove an analog of Theorem 2.14 for the symmetric $G$-metrics.

\section{Extended by Balk Metrics and $G$-metrics}

The domain of $\tau^{3}$ (see formula (1.4)) is the set $X^{3}=X \times X \times X$. Different generalized metrics with this domain were considered at least since $60 \mathrm{~s}$ of the last century $[14,15,6]$. The so-called $G$-metric (see Definition 1.5 ) is among the most important from these generalizations. The $G$-metric was introduced by Mustafa and Sims $[18,20]$ and has applications in the fixed point theory.

In the current section we, in particular, show that the functions $\tau^{3}: X^{3} \rightarrow \mathbb{R}$ generated by increasing extended by Balk metrics $\tau: \mathcal{F}(X) \rightarrow \mathbb{R}$ are symmetric (in the sense of Definition $1.6 G$-metrics on $X$.

Lemma 3.1. Let $X \neq \varnothing$ and let $\tau: \mathcal{F}(X) \rightarrow \mathbb{R}$ be an increasing extended by Balk metric. Then $\tau^{3}$ is a symmetric $G$-metric on $X$.

Proof. By Definition (1.3) $\tau^{3}$ is a symmetric $G$-metric if and only if the equality $\tau^{3}(x, y, y)=\tau^{3}(y, x, x)$ holds for all $x, y \in X$. This equality immediately follows from (1.4) and (1.5).

Let us check conditions (i)-(v) of Definition 1.5.

(i) For every $x$ the equality $\tau^{3}(x, x, x)=0$ follows from (1.1). 
(ii) The inequality $\tau^{3}(x, x, y)>0$ for $x \neq y$ follows from the equality $\tau^{3}(x, x, y)=$ $\tau^{2}(x, y)$ and the fact that $\tau^{2}$ is a metric on $X$ (see Proposition 2.1).

(iii) The inequality $\tau^{3}(x, x, y) \leqslant \tau^{3}(x, y, z)$ follows because $\tau$ is increasing.

(iv) The arguments of the function $\tau$ on the right-hand side of equality (1.4) are sets, that automatically gives the invariance of $\tau^{3}$ with respect to the permutations of arguments.

(v) We must prove the inequality

$$
\tau^{3}(x, y, z) \leqslant \tau^{3}(x, a, a)+\tau^{3}(a, y, z)
$$

for all $x, y, z, a \in X$. The inequality holds if $x=y=z$ since $\tau^{3}$ is a nonnegative function and $\tau(x, x, x)=0$. Now let $x \neq y \neq z \neq x$. Substituting $A=\{x\}, B=\{y, z\}, C=\{a\}$ in (1.2) we obtain

$$
\tau^{3}(x, y, z)=\tau(A \cup B) \leqslant \tau(A \cup C)+\tau(B \cup C)=\tau^{3}(x, a, a)+\tau^{3}(a, y, z) .
$$

If $y=z$, inequality (3.1) is equivalent to the triangle inequality

$$
\tau^{2}(x, y) \leqslant \tau^{2}(x, a)+\tau^{2}(a, y),
$$

that was proved in Proposition 2.1. Let $x=z$. Then (3.1) can be written as

$$
\tau^{3}(x, x, y) \leqslant \tau^{3}(x, a, a)+\tau^{3}(a, y, x) .
$$

Since $\tau$ is increasing, the inequality $\tau^{3}(a, y, x) \geqslant \tau^{3}(a, y, y)$ holds. Therefore, it is sufficient to check $\tau^{3}(x, x, y) \leqslant \tau^{3}(x, a, a)+\tau^{3}(a, y, y)$ which again reduces to the triangle inequality for $\tau^{2}$. It remains to consider the case where $x=y$. With this assumption (3.1) we get

$$
\tau^{3}(x, x, z) \leqslant \tau^{3}(x, a, a)+\tau^{3}(a, x, z) .
$$

Again from the increase of $\tau$ we obtain $\tau^{3}(a, x, z) \geqslant \tau^{3}(a, z, z)$. Hence it suffices to prove the inequality $\tau^{3}(x, x, z) \leqslant \tau^{3}(x, a, a)+\tau^{3}(a, z, z)$, which also follows from the triangle inequality.

Now we want to prove the converse of Lemma 3.1. To do this it suffices for given symmetric $G$-metric $\Phi: X^{3} \rightarrow \mathbb{R}$ to construct an increasing extended by Balk metric $\tau: \mathcal{F}(X) \rightarrow \mathbb{R}$ such that

$$
\Phi\left(x_{1}, x_{2}, x_{3}\right)=\tau\left(\operatorname{Im}\left(x_{1}, x_{2}, x_{3}\right)\right), \quad\left(x_{1}, x_{2}, x_{3}\right) \in X^{3},
$$

where $\operatorname{Im}\left(x_{1}, x_{2}, x_{3}\right)$ was defined by relation (1.5). We will carry out this construction in two steps. 
- For given $G$-metric $\Phi: X^{3} \rightarrow \mathbb{R}$ we first find an increasing mapping $\tilde{\tau}$ : $\mathcal{F}^{3}(X) \rightarrow \mathbb{R}, \mathcal{F}^{3}(X)=\{A \in \mathcal{F}(X):|A| \leqslant 3\}$, which satisfies (3.4) and (1.1), (1.2) with $\tau=\tilde{\tau}$. (This is almost what we need but the domain of $\tilde{\tau}$ is $\left.\mathcal{F}^{3}(X)\right)$.

- Second, we expand $\tilde{\tau}$ to an increasing extended by Balk metric $\tau: \mathcal{F}(X) \rightarrow \mathbb{R}$.

Lemma 3.2. Let $X \neq \varnothing$. The following statements are equivalent for every function $G: X^{3} \rightarrow \mathbb{R}$.

(i) G satisfies condition (iv) of Definition 1.5 and is symmetric in the sense that

$$
G(x, y, y)=G(y, x, x)
$$

for all $x, y \in X$.

(ii) There is a mapping $\tilde{\tau}: \mathcal{F}^{3}(X) \rightarrow \mathbb{R}$ such that equality (3.4) holds for every $\left(x_{1}, x_{2}, x_{3}\right) \in X^{3}$ with $\Phi=G$ and $\tau=\tilde{\tau}$.

Proof. The implication (ii) $\Rightarrow$ (i) has already been proved in the proof of Lemma 3.1.

Let us verify the implication (i) $\Rightarrow$ (ii). Suppose (i) holds. It is sufficient to check that the equality

$$
\operatorname{Im}\left(x_{1}, x_{2}, x_{3}\right)=\operatorname{Im}\left(y_{1}, y_{2}, y_{3}\right)
$$

implies

$$
G\left(x_{1}, x_{2}, x_{3}\right)=G\left(y_{1}, y_{2}, y_{3}\right) .
$$

Let (3.6) hold. If $\left|\operatorname{Im}\left(x_{1}, x_{2}, x_{3}\right)\right|=1$, then there is $x \in X$ such that $x_{i}=x=y_{i}$ for every $i \in\{1,2,3\}$. In this case (3.7) transforms to the trivial equality $G(x, x, x)=$ $G(x, x, x)$. If $\left|\operatorname{Im}\left(x_{1}, x_{2}, x_{3}\right)\right|=3$, then (3.7) follows from the invariance of $G$ with respect to the permutations of arguments. For the case $\left|\operatorname{Im}\left(x_{1}, x_{2}, x_{3}\right)\right|=2$ there are $x, y \in X$ for which the triple $\left(x_{1}, x_{2}, x_{3}\right)$ coincides with one of the triples $(x, x, y),(x, y, x),(y, x, x),(y, y, x),(y, x, y),(x, y, y)$. The same holds for $\left(y_{1}, y_{2}, y_{3}\right)$ also. Now to prove (3.7) we can use (3.5) and the invariance of $G$ with respect to the permutations of arguments.

Remark 3.3. Since the mapping $X^{3} \ni\left(x_{1}, x_{2}, x_{3}\right) \mapsto \operatorname{Im}\left(x_{1}, x_{2}, x_{3}\right) \in \mathcal{F}^{3}(X)$ is surjective, the existence of $\tilde{\tau}: \mathcal{F}^{3}(X) \rightarrow \mathbb{R}$ for which the equality

$$
G\left(x_{1}, x_{2}, x_{3}\right)=\tilde{\tau}\left(\operatorname{Im}\left(x_{1}, x_{2}, x_{3}\right)\right)
$$

holds for every $\left(x_{1}, x_{2}, x_{3}\right) \in X^{3}$ implies the uniqueness of $\tilde{\tau}$.

Lemma 3.4. Let $X \neq \varnothing$ and let $G: X^{3} \rightarrow \mathbb{R}$ be a symmetric $G$-metric. Then there is an increasing mapping $\tilde{\tau}: \mathcal{F}^{3}(X) \rightarrow \mathbb{R}$ such that: equality (3.8) holds for every $\left(x_{1}, x_{2}, x_{3}\right) \in X^{3}$; equivalence (1.1) holds with $\tilde{\tau}=\tau$ for every $A \in \mathcal{F}^{3}(X)$; inequality (1.2) holds with $\tilde{\tau}=\tau$ for all $A \cup B, A \cup C, B \cup C \in \mathcal{F}^{3}(X)$. 
Proof. The existence of $\tilde{\tau}: \mathcal{F}^{3}(X) \rightarrow \mathbb{R}$ which satisfies (3.8) for $\left(x_{1}, x_{2}, x_{3}\right) \in X^{3}$ has already proved in Lemma 3.2. The increase of $\tilde{\tau}$ and the equivalence

$$
(\tilde{\tau}(A)=0) \Leftrightarrow(|A|=1)
$$

follow from conditions (i) - (iii) of Definition 1.5 and equality (3.8). We must prove only the inequality

$$
\tilde{\tau}(A \cup B) \leqslant \tilde{\tau}(A \cup C)+\tilde{\tau}(B \cup C)
$$

for $A \cup B, A \cup C, B \cup C \in \mathcal{F}^{3}(X)$.

Note that (3.9) is trivial if $|A \cup B|=1$ because in this case $\tilde{\tau}(A \cup B)=0$ holds. So we can suppose $|A \cup B|=2$ or $|A \cup B|=3$. Since $\tilde{\tau}$ is increasing, it suffices to prove (3.9) for $C=\{a\}$ where $a$ is an arbitrary point of $X$.

Let $|A \cup B|=2$. If, in addition, we have $|A|=2$, then

$$
A \cup B=A \subseteq A \cup C .
$$

Hence using the nonnegativity of $G$ (see Remark 1.7) and the increase of $\tilde{\tau}$ we obtain (3.9). If $|B|=2$, then the proof is similar. Now let $A=\{x\}, B=\{y\}$ and $x \neq y$. Then

$$
\begin{gathered}
\tilde{\tau}(A \cup B)=G(x, y, y), \quad \tilde{\tau}(A \cup C)=G(x, a, a), \\
\tilde{\tau}(B \cup C)=G(y, a, a)=G(a, y, y) .
\end{gathered}
$$

Putting $z=y$ in condition (v) of Definition 1.5 we find

$$
G(x, y, y) \leqslant G(x, a, a)+G(a, y, y) .
$$

This inequality and (3.11) give (3.9).

Suppose $|A \cup B|=3$. If $\max (|A|,|B|)=3$, then we have (3.10) or $A \cup B \subseteq B \cup C$. Hence using the increase of $\tau^{*}$ we get (3.9). If $|A|=2$ and $|B|=2$, then there are some distinct $x, y, z \in X$ for which $A=\{x, y\}$ and $B=\{y, z\}$. Consequently $\tilde{\tau}(A \cup B)=G(x, y, z), \tilde{\tau}(A \cup C)=G(x, y, a)$ and $\tilde{\tau}(B \cup C)=G(y, z, a)$. Inequality (3.9) can be rewritten as

$$
G(x, y, z) \leqslant G(x, y, a)+G(y, z, a) .
$$

Using condition (iii) of Definition 1.5 and the symmetry of $G$ we obtain the inequality

$$
G(x, a, a) \leqslant G(x, y, a)
$$

for all $x, y, a \in X$. Now (3.13) and condition (v) of Definition 1.5 imply (3.12). To complete the proof it remains to consider the next alternative

$$
\text { either }|A|=1 \text { and }|B|=2 \quad \text { or } \quad|B|=1 \text { and }|A|=2 .
$$


By the symmetry of the occurrences of $A$ and $B$ in (3.9) it suffices to consider the first case. Putting $A=\{x\}$ and $B=\{y, z\}$ and expressing $\tilde{\tau}$ via $G$, we get from (3.9) to the inequality $G(x, y, z) \leqslant G(x, a, a)+G(a, y, z)$. Condition $(v)$ of Definition 1.5 claims the validity of the last inequality.

In accordance with our plan it remains expand the function $\tilde{\tau}: \mathcal{F}^{3}(X) \rightarrow \mathbb{R}$ to an increasing extended by Balk metric $\tau: \mathcal{F}(X) \rightarrow \mathbb{R}$. It is easy enough to do for all increasing $\tilde{\tau}: \mathcal{F}^{k}(X) \rightarrow \mathbb{R}, \mathcal{F}^{k}(X)=\{A \in \mathcal{F}(X):|A| \leqslant k\}$ with an arbitrary integer $k \geqslant 2$.

For $A \in \mathcal{F}(X)$ and $\tilde{\tau}: \mathcal{F}^{k}(X) \rightarrow \mathbb{R}, k \geqslant 1$ we set

$$
\operatorname{diam}_{\tilde{\tau}}(A):=\max \left\{\tilde{\tau}(B): B \subseteq A, B \in \mathcal{F}^{k}(X)\right\},
$$

c.f. formula (1.6).

Proposition 3.5. Let $X \neq \varnothing$, let $k \geqslant 2$ be an integer number and let $\tilde{\tau}: \mathcal{F}^{k}(X) \rightarrow$ $\mathbb{R}$ be an increasing mapping such that the equivalence

$$
(\tilde{\tau}(A)=0) \Leftrightarrow(|A|=1)
$$

holds for each $A \in \mathcal{F}^{k}(X)$ and the inequality

$$
\tilde{\tau}(A \cup B) \leqslant \tilde{\tau}(A \cup C)+\tilde{\tau}(B \cup C)
$$

holds as soon as $A \cup B, A \cup C, B \cup C \in \mathcal{F}^{k}(X)$. Then the function

$$
\tau: \mathcal{F}(X) \rightarrow \mathbb{R}, \quad \tau(A)=\operatorname{diam}_{\tilde{\tau}}(A), \quad A \in \mathcal{F}(X)
$$

is an increasing extended by Balk metric such that

$$
\tau^{k}\left(x_{1}, \ldots, x_{k}\right)=\tilde{\tau}\left(\operatorname{Im}\left(x_{1}, \ldots, x_{k}\right)\right) \quad \text { for } \quad\left(x_{1}, \ldots, x_{k}\right) \in X^{k} .
$$

Proof. The increase of $\tau$ follows directly from equality (3.14). This equality and the increase of $\tilde{\tau}$ give also equality (3.18). Using (3.14) it is easy to prove (3.15) for every $A \in \mathcal{F}(X)$. It remains to show that the inequality

$$
\tau(A \cup B) \leqslant \tau(A \cup C)+\tau(B \cup C),
$$

holds for all $A, B, C \in \mathcal{F}(X)$.

Let $A, B$ and $C$ be arbitrary elements of $\mathcal{F}(X)$. Let us choose an element $D \in \mathcal{F}^{k}(X)$ such that

$$
D \subseteq A \cup B \text { and } \tau(A \cup B)=\tilde{\tau}(D) .
$$

If $D \subseteq A$ or $D \subseteq B$, then by increase of $\tau$ we have $\tau(D) \leqslant \tau(A) \leqslant \tau(A \cup C)$ or, respectively, $\tau(D) \leqslant \tau(B) \leqslant \tau(B \cup C)$. These inequalities together with (3.20) give (3.19). Thus we can assume that

$$
D \backslash A \neq \varnothing \neq D \backslash B .
$$


Set $A^{\prime}:=A \cap D, B^{\prime}:=B \cap D$. Then we have

$$
D=D \cap(A \cup B)=A^{\prime} \cup B^{\prime} .
$$

Condition (3.21) and the inequality $|A| \leqslant k$ give the inequalities

$$
\left|A^{\prime}\right| \leqslant k-1, \quad \text { and } \quad\left|B^{\prime}\right| \leqslant k-1 .
$$

Using (3.20) and (3.22) we write (3.19) in the form

$$
\tau\left(A^{\prime} \cup B^{\prime}\right) \leqslant \tau(A \cup C)+\tau(B \cup C) .
$$

Since $\tau$ is increasing, $A^{\prime} \subseteq A$ and $B^{\prime} \subseteq B$, it suffices to check the inequality

$$
\tau\left(A^{\prime} \cup B^{\prime}\right) \leqslant \tau\left(A^{\prime} \cup C\right)+\tau\left(B^{\prime} \cup C\right) .
$$

Let $C^{\prime}=\{c\}$ where $c \in C$. Since $\tau$ is increasing, we have

$$
\tau\left(A^{\prime} \cup C\right)+\tau\left(B^{\prime} \cup C\right) \geqslant \tau\left(A^{\prime} \cup C^{\prime}\right)+\tau\left(B^{\prime} \cup C^{\prime}\right) .
$$

Therefore it is sufficient to show that

$$
\tau\left(A^{\prime} \cup B^{\prime}\right) \leqslant \tau\left(A^{\prime} \cup C^{\prime}\right)+\tau\left(B^{\prime} \cup C^{\prime}\right) .
$$

To prove (3.24) note that (3.23) implies that

$$
\left|A^{\prime} \cup C^{\prime}\right| \leqslant\left|A^{\prime}\right|+\left|C^{\prime}\right| \leqslant(k-1)+1=k,
$$

and, similarly that $\left|B^{\prime} \cup C^{\prime}\right| \leqslant k$. Thus $A^{\prime} \cup C^{\prime}, B^{\prime} \cup C^{\prime} \in \mathcal{F}^{k}(X)$. In addition we have $A^{\prime} \cup B^{\prime}=D \in \mathcal{F}^{k}(X)$. Now using (3.18) we can rewrite (3.24) in the form $\tilde{\tau}\left(A^{\prime} \cup B^{\prime}\right) \leqslant \tilde{\tau}\left(A^{\prime} \cup C^{\prime}\right)+\tilde{\tau}\left(B^{\prime} \cup C^{\prime}\right)$ that holds by $(3.16)$.

Thus the function $\tau$ defined on $\mathcal{F}(X)$ by formula (3.17) has all properties of extended by Balk metric.

Remark 3.6. Proposition 3.5 is false for $k=1$. In this case we have $\operatorname{diam}_{\tilde{\tau}}(A)=0$ for every $A \in \mathcal{F}(X)$.

Theorem 3.7. Let $X \neq \varnothing$. The following statements are equivalent for every function $G: X^{3} \rightarrow \mathbb{R}$.

(i) $G$ is a symmetric $G$-metric in the sense of Definition 1.5.

(ii) There is an increasing extended by Balk metric $\tau: \mathcal{F}(X) \rightarrow \mathbb{R}$ such that $\tau^{3}=G$.

Proof. The implication (ii) $\Rightarrow$ (i) was obtained in Lemma 3.1. Let us prove the implication (i) $\Rightarrow$ (ii). Suppose (i) holds. By Lemma 3.2 there is $\tilde{\tau}: \mathcal{F}^{3}(X) \rightarrow \mathbb{R}$ such that $G\left(x_{1}, x_{2}, x_{3}\right)=\tilde{\tau}\left(\operatorname{Im}\left(x_{1}, x_{2}, x_{3}\right)\right)$ for every $\left(x_{1}, x_{2}, x_{3}\right) \in X^{3}$. Using Lemma 3.4 we get the equivalence $(\tilde{\tau}(A)=0) \Leftrightarrow(|A|=1)$ for every $A \in \mathcal{F}^{3}(X)$ 
and the inequality $\tilde{\tau}(A \cup B) \leqslant \tilde{\tau}(A \cup C)+\tilde{\tau}(B \cup C)$ for $A \cup B, A \cup C, B \cup C \in \mathcal{F}^{3}(X)$. By Proposition 3.5 there is an increasing extended by Balk metric $\tau: \mathcal{F}(X) \rightarrow \mathbb{R}$ such that $\left.\right|_{\mathcal{F}^{3}(X)}=\tilde{\tau}$. The implication (i) $\Rightarrow$ (ii) is proved.

The next theorem is an analog of Theorem 2.14.

Theorem 3.8. Let $\tau: \mathcal{F}(X) \rightarrow \mathbb{R}$ be an extended by Balk metric on $X \neq \varnothing$. The following statements are equivalent.

(i) There is a function $G: X^{3} \rightarrow \mathbb{R}$ such that the equality

$$
\tau(A)=\max \{G(x, y, z): x, y, z \in A\}
$$

holds for every $A \in \mathcal{F}(X)$.

(ii) There is a symmetric G-metric $G: X^{3} \rightarrow \mathbb{R}$ such that equality (3.25) holds for every $A \in \mathcal{F}(X)$.

(iii) For every $A \in \mathcal{F}(X)$ the equality (3.25) holds with $G=\tau^{3}$.

(iv) $\tau$ is 3-increasing and 3-weakly decreasing.

(v) $\tau$ is increasing and 3-weakly decreasing.

Proof. If (iii) holds, then $\tau$ is increasing. Then, by Lemma 3.1, $\tau^{3}$ is symmetric $G$-metric. Therefore the implication (iii) $\Rightarrow$ (ii) is true. The implication (ii) $\Rightarrow$ (i) is obvious. The implication (i) $\Rightarrow(\mathrm{v})$ follows directly from definitions. To complete the proof it remains to note that Theorem 2.12 gives (v) $\Leftrightarrow$ (iv) and (iv) $\Leftrightarrow$ (iii).

\section{Extended Metrics on Pretangent Spaces}

Let $(X, d)$ be a metric space with a metric $d$. The infinitesimal geometry of the space $X$ can be investigated by constructing of metric spaces that, in some sense, are tangent to $X$. If $X$ is equipped with an additional structure, then the question arises of the lifting this structure on the tangent spaces. More specifically, let $\tau: \mathcal{F}(X) \rightarrow \mathbb{R}$ be an extended by Balk metric and let $(\Omega, \rho)$ be a tangent space to a metric space $(X, d)$. Suppose $\tau$ is compatible with $d$.

How to build an extended by Balk metric which is compatible with the metric $\rho$ ?

The answer to this question depends on the construction of the tangent space $(\Omega, \rho)$. Today there are several approaches to construct tangent spaces to metric spaces. Probably, the most famous of these are the Gromov-Hausdorff convergence and the ultra-convergence. The sequential approach to the construction of "pretangent" and "tangent" spaces was proposed in [12] and developed in [10, 1, 5, 4, 7, 9].

To construct Balk's extended metrics on pretangent spaces we will use ultrafilters on $\mathbb{N}$. Recall the necessary definitions.

Let $X$ be a nonempty set and let $\mathfrak{B}(X)$ be the set of all its subsets. A set $\mathfrak{U} \subseteq \mathfrak{B}(X)$ is called a filter on $X$ if $\varnothing \notin \mathfrak{U} \neq \varnothing$ and the implication

$$
(A \in \mathfrak{U} \& B \in \mathfrak{U}) \Rightarrow(A \cap B \in \mathfrak{U})) \quad \text { and } \quad(A \supseteq B \& B \in \mathfrak{U}) \Rightarrow(A \in \mathfrak{U}))
$$


hold for all $A, B \subseteq X$.

A filter $\mathfrak{U}$ on $X$ is called an ultrafilter if the implication $(\mathcal{P} \supseteq \mathfrak{U}) \Rightarrow(\mathfrak{U}=\mathcal{P})$ holds for every filter $\mathcal{P}$ on $X$. An ultrafilter $\mathfrak{U}$ on $X$ is called trivial if there is a point $x_{0} \in X$ such that $(A \in \mathfrak{U}) \Leftrightarrow\left(x_{0} \in A\right)$ for $A \in \mathfrak{B}(X)$. Otherwise $\mathfrak{U}$ is a nontrivial ultrafilter. Let $\mathfrak{U}$ be a filter on $X$. A mapping $\Phi: X \rightarrow \mathbb{R}$ converges to a point $t \in \mathbb{R}$ by the filter $\mathfrak{U}$, symbolically $\mathfrak{U}-\lim \Phi(x)=t$, if

$$
\{x \in X:|\Phi(x)-t|<\varepsilon\} \in \mathfrak{U}
$$

for every $\varepsilon>0$.

Example 4.1. If $\mathcal{M}$ is the Frechet filter on $\mathbb{N}$, (is the family of subsets of $\mathbb{N}$ with finite complements) and $\left(x_{n}\right)_{n \in \mathbb{N}}$ is a sequence of real numbers, then the limit $\lim _{n \rightarrow \infty} x_{n}$ exists if and only if there is $\mathcal{M}-\lim x_{n}$. In this case $\lim _{n \rightarrow \infty} x_{n}=\mathcal{M}-\lim x_{n}$.

We shall use the following properties of the nontrivial ultrafilters $\mathfrak{U}$ on $\mathbb{N}$.

$\left(\mathrm{i}_{1}\right)$ Every bounded sequence $\left(x_{n}\right)_{n \in \mathbb{N}}, x_{n} \in \mathbb{R}$, has $\mathfrak{U}-\lim x_{n}$;

(i $\left.i_{2}\right)$ If $\left(x_{n}\right)_{n \in \mathbb{N}}$ converges in the usual sense, then $\lim _{n \rightarrow \infty} x_{n}=\mathfrak{U}-\lim x_{n}$;

$\left(\mathrm{i}_{3}\right)$ The relations $\mathfrak{U}-\lim c x_{n}=c\left(\mathfrak{U}-\lim x_{n}\right)$ and

$$
\mathfrak{U}-\lim \left(x_{n}+y_{n}\right)=\left(\mathfrak{U}-\lim x_{n}\right)+\left(\mathfrak{U}-\lim y_{n}\right)
$$

hold for every $c \in \mathbb{R}$ and $\left(x_{n}\right)_{n \in \mathbb{N}},\left(y_{n}\right)_{n \in \mathbb{N}}$ which have $\mathfrak{U}$-limits;

$\left(i_{4}\right)$ If $\left(x_{n}\right)_{n \in \mathbb{N}}$ is $\mathfrak{U}$-convergent and $\lim _{n \rightarrow \infty}\left(x_{n}-y_{n}\right)=0$, then $\left(y_{n}\right)_{n \in \mathbb{N}}$ is $\mathfrak{U}$ convergent and $\mathfrak{U}-\lim x_{n}=\mathfrak{U}-\lim y_{n}$.

The above is a trivial modification of Problem 19 from Chapter 17 [17].

Lemma 4.2. Let $\mathfrak{U}$ be a nontrivial ultrafilter on $\mathbb{N}$. Then for every bounded sequence $\left(x_{m}\right)_{m \in \mathbb{N}}, x_{m} \in \mathbb{R}$ its $\mathfrak{U}$-limit coincides with a limit point of this sequence. Conversely, if $t$ is a limit point of $\left(x_{m}\right)_{m \in \mathbb{N}}$, then there is a nontrivial ultrafilter $\mathfrak{U}$ on $\mathbb{N}$ such that $\mathfrak{U}-\lim x_{m}=t$.

Proof. The first statement of the lemma follows from the definition of the limit points and formula (4.1) if put $X=\mathbb{N}$ and $\Phi(n)=x_{n}$ in this formula and take into account that all elements of nontrivial ultrafilter on $\mathbb{N}$ are infinite subsets of $\mathbb{N}$.

To prove the second statement, note that for every limit point $a$ of the sequence $\left(x_{m}\right)_{m \in \mathbb{N}}$ there is an infinite $A \subseteq \mathbb{N}$ such that $\lim _{\substack{m \rightarrow \infty \\ m \in A}} x_{m}=a$. Choose an ultrafilter $\mathfrak{U}$ on $\mathbb{N}$ for which $A \in \mathfrak{U}$. Now using property $\left(i_{1}\right)$ we obtain $a=\mathfrak{U}-\lim x_{m}$.

Let $(X, d)$ be a metric space, $X \neq \varnothing$, and let $\tau: \mathcal{F}(X) \rightarrow \mathbb{R}$ be a compatible with $d$ extended by Balk metric. Let $\left\{\alpha_{1}, \ldots, \alpha_{n}\right\}$ be a finite nonempty subset of pretangent space $\Omega_{p, \tilde{r}}^{X}$ and $\tilde{X}_{p, \tilde{r}}$ be a maximal self-stable subset of $\tilde{X}_{p}$ which corresponds $\Omega_{p, \tilde{r}}^{X}$. Denote by $\pi$ the projection $\tilde{X}_{p, \tilde{r}}$ on $\Omega_{p, \tilde{r}}^{X}$, i.e. if $\tilde{x} \in \tilde{X}_{p, \tilde{r}}$ then 
$\pi(\tilde{x})=\left\{\tilde{y} \in \tilde{X}_{p, \tilde{r}}: \tilde{d}_{\tilde{r}}(\tilde{x}, \tilde{y})=0\right\}$. (See formula (1.7)). Choose $\tilde{x}^{i}=\left(x_{m}^{i}\right)_{m \in \mathbb{N}}$, $i=1, \ldots, n$ from $\tilde{X}_{p, \tilde{r}}$ such that $\pi\left(\tilde{x}^{i}\right)=\alpha_{i}, \quad i=1, \ldots, n$. Put

$$
\mathcal{X}_{\tau}\left(\left\{\alpha_{1}, \ldots, \alpha_{n}\right\}\right):=\mathfrak{U}-\lim \frac{\tau\left(\operatorname{Im}\left(x_{m}^{1}, \ldots, x_{m}^{n}\right)\right)}{r_{m}},
$$

where $\operatorname{Im}\left(x_{m}^{1}, \ldots, x_{m}^{n}\right)$ is the set whose elements are the $m$-th terms of the sequences $\left(x_{m}^{i}\right)_{m \in \mathbb{N}}, i=1, \ldots, n, r_{m}$ is $m$-th term of normalizing sequence $\tilde{r}$ and $\mathfrak{U}$ is a nontrivial ultrafilter on $\mathbb{N}$.

Theorem 4.3. Let $(X, d, p)$ be a metric space with a marked point $p$ and $\tau$ : $\mathcal{F}(X) \rightarrow \mathbb{R}$ be an extended by Balk metric. If $\tau$ is compatible with $d$, then for every pretangent space $\left(\Omega_{p, \tilde{r}}^{X}, \rho\right)$ and every nontrivial ultrafilter $\mathfrak{U}$ on $\mathbb{N}$ the mapping

$$
\mathcal{F}\left(\Omega_{p, \tilde{r}}^{X}\right) \ni A \mapsto X_{\tau}(A)
$$

is correctly defined extended by Balk metric which is compatible with the metric $\rho$.

To prove this theorem we need the next lemma.

Lemma 4.4. Let $(X, d)$ be a nonempty metric space and $\tau: \mathcal{F}(X) \rightarrow \mathbb{R}$ be an extended by Balk metric. If $\tau$ is compatible with $d$, then the inequalities

$$
\tau\left(\left\{x_{1}, x_{2}, \ldots, x_{n}\right\}\right) \leqslant d\left(x_{1}, x_{2}\right)+\ldots+d\left(x_{n-1}, x_{n}\right),
$$

and

$$
\left|\tau\left(\left\{x_{1}, \ldots, x_{n}\right\}\right)-\tau\left(\left\{x_{1}^{\prime}, \ldots, x_{n}^{\prime}\right\}\right)\right| \leqslant \sum_{i=1}^{n} d\left(x_{i}, x_{i}^{\prime}\right),
$$

hold for every integer $n \geqslant 1$. Here $\left\{x_{1}, \ldots, x_{n}\right\}$ and $\left\{x_{1}^{\prime}, \ldots, x_{n}^{\prime}\right\}$ are arbitrary $n$ elements subsets of the set $X$.

Proof. Without loss of generality we can suppose that $n \geqslant 2$. Let $\left\{x_{1}, \ldots, x_{n}\right\} \in$ $\mathcal{F}(X)$. Using (2.1) with $B=\left\{x_{n}\right\}, C=\left\{x_{n-1}\right\}$ and $A=\left\{x_{1}, \ldots, x_{n-1}\right\}$ we find

$$
\begin{gathered}
\tau\left(\left\{x_{1}, \ldots, x_{n}\right\}\right) \leqslant \tau\left(\left\{x_{1}, \ldots, x_{n-1}\right\}\right)+\tau\left(\left\{x_{n-1}, x_{n}\right\}\right) \\
\leqslant \tau\left(\left\{x_{1}, \ldots, x_{n-1}\right\}\right)+d\left(x_{n-1}, x_{n}\right) .
\end{gathered}
$$

Repeating this procedure we obtain inequality (4.3).

Let us check (4.4). To this end note that

$$
\begin{gathered}
\left|\tau\left(\left\{x_{1}, \ldots, x_{n}\right\}\right)-\tau\left(\left\{x_{1}^{\prime}, \ldots, x_{n}^{\prime}\right\}\right)\right| \\
\leqslant\left|\tau\left(\operatorname{Im}\left(x_{1}, x_{2} \ldots, x_{n}\right)\right)-\tau\left(\operatorname{Im}\left(x_{1}^{\prime}, x_{2} \ldots, x_{n}\right)\right)\right| \\
+\left|\tau\left(\operatorname{Im}\left(x_{1}^{\prime}, x_{2} \ldots, x_{n}\right)\right)-\tau\left(\operatorname{Im}\left(x_{1}^{\prime}, x_{2}^{\prime}, x_{3} \ldots, x_{n}\right)\right)\right| \ldots \\
+\left|\tau\left(\operatorname{Im}\left(x_{1}^{\prime}, x_{2}^{\prime} \ldots, x_{n-1}^{\prime}, x_{n}\right)\right)-\tau\left(\operatorname{Im}\left(x_{1}^{\prime}, \ldots, x_{n-1}^{\prime}, x_{n}^{\prime}\right)\right)\right| .
\end{gathered}
$$


The sets, which are the arguments of the function $\tau$ under the signs of the absolute value on the right-hand side of the last inequality, differ from each other by no more than one element. Therefore, it suffices to verify the inequality

$$
\left|\tau\left(\operatorname{Im}\left(x_{1}^{\prime}, \ldots, x_{n-1}^{\prime}, x_{n}\right)\right)-\tau\left(\operatorname{Im}\left(x_{1}^{\prime}, \ldots, x_{n-1}^{\prime}, x_{n}^{\prime}\right)\right)\right| \leqslant d\left(x_{n}, x_{n}^{\prime}\right) .
$$

Without loss of generality we can suppose that

$$
\tau\left(\operatorname{Im}\left(x_{1}^{\prime}, \ldots, x_{n-1}^{\prime}, x_{n}\right)\right) \geqslant \tau\left(\operatorname{Im}\left(x_{1}^{\prime}, \ldots, x_{n-1}^{\prime}, x_{n}^{\prime}\right)\right) .
$$

Using inequality (1.2) with $A=\left\{x_{1}^{\prime}, \ldots, x_{n-1}^{\prime}\right\}, B=\left\{x_{n}\right\}, C=\left\{x_{n}^{\prime}\right\}$ we get $A \cup B=\operatorname{Im}\left(x_{1}^{\prime}, \ldots, x_{n-1}^{\prime}, x_{n}\right), A \cup C=\operatorname{Im}\left(x_{1}^{\prime}, \ldots, x_{n-1}^{\prime}, x_{n}^{\prime}\right), B \cup C=\operatorname{Im}\left(x_{n}, x_{n}^{\prime}\right)$ and

$$
\tau\left(\operatorname{Im}\left(x_{1}^{\prime}, \ldots, x_{n-1}^{\prime}, x_{n}\right)\right)-\tau\left(\operatorname{Im}\left(x_{1}^{\prime}, \ldots, x_{n-1}^{\prime}, x_{n}^{\prime}\right)\right) \leqslant \tau\left(\left\{x_{n}, x_{n}^{\prime}\right\}\right)=d\left(x_{n}, x_{n}^{\prime}\right) .
$$

The last inequality together with (4.6) gives (4.5).

Lemma 4.5. Let $(X, d)$ be a nonempty metric space, let $\tau: \mathcal{F}(X) \rightarrow \mathbb{R}$ be an extended by Balk metric and let $K \in \mathcal{F}(X)$. If $\tau$ is compatible with $d$, then the inequality

$$
\tau(K) \geqslant \frac{1}{2} d(x, y)
$$

holds for all $x, y \in K$.

Proof. Let $A=\{x\}, B=\{y\}$ and $C=K$. Then by inequality (1.2) we have

$$
d(x, y)=\tau(A \cup B) \leqslant \tau(A \cup K)+\tau(B \cup K)=2 \tau(K) .
$$

The proof of the Theorem 4.3. Let us check the existence of the finite $\mathfrak{U}$-limit on the right-hand side of (4.2). According to property $\left(\mathrm{i}_{1}\right)$ of the ultrafilters it suffices to prove the inequality

$$
\limsup _{m \rightarrow \infty} \frac{\tau\left(\operatorname{Im}\left(x_{m}^{1}, \ldots, x_{m}^{n}\right)\right)}{r_{m}}<\infty .
$$

Using (1.7), (1.8) and (4.3) we find

$$
\begin{gathered}
\limsup _{m \rightarrow \infty} \frac{\tau\left(\operatorname{Im}\left(x_{m}^{1}, \ldots, x_{m}^{n}\right)\right)}{r_{m}} \leqslant \lim _{m \rightarrow \infty} \frac{d\left(x_{m}^{1}, x_{m}^{2}\right)}{r_{m}} \\
+\lim _{m \rightarrow \infty} \frac{d\left(x_{m}^{2}, x_{m}^{3}\right)}{r_{m}}+\ldots+\lim _{m \rightarrow \infty} \frac{d\left(x_{m}^{n-1}, x_{m}^{n}\right)}{r_{m}}=\sum_{i=1}^{n-1} \rho\left(\alpha_{i}, \alpha_{i+1}\right) .
\end{gathered}
$$

Inequality (4.8) follows. 
Let us make sure that the value of $\mathcal{X}_{\tau}\left(\left\{\alpha_{1}, \ldots, \alpha_{n}\right\}\right)$ given in formula (4.2) does not depend of the choice of $\tilde{x}^{i}, i=1, \ldots, n$. Let $\tilde{y}^{i}=\left(y_{m}^{i}\right)$ be some elements of the set $\tilde{X}_{p, \tilde{r}}$ such that $\pi\left(\tilde{y}^{i}\right)=\pi\left(\tilde{x}^{i}\right)=\alpha_{i}, i=1, \ldots, n$.

By (4.4) we have

$$
\begin{gathered}
\limsup _{m \rightarrow \infty} \frac{\tau\left(\operatorname{Im}\left(x_{m}^{1}, \ldots, x_{m}^{n}\right)\right)-\tau\left(\operatorname{Im}\left(y_{m}^{1}, \ldots, y_{m}^{n}\right)\right)}{r_{m}} \\
\leqslant \sum_{i=1}^{n}\left(\lim _{m \rightarrow \infty} \frac{d\left(x_{m}^{i}, y_{m}^{i}\right)}{r_{m}}\right)=\sum_{i=1}^{n} \rho\left(\alpha_{i}, \alpha_{i}\right)=0 .
\end{gathered}
$$

The wanted independence follows from $\left(i_{4}\right)$.

Let us verify that $X_{\tau}: \mathcal{F}\left(\Omega_{p, \tilde{r}}^{X}\right) \rightarrow \mathbb{R}$ has the characteristic properties of extended metric i.e.,

$$
\left(X_{\tau}(A)=0\right) \Leftrightarrow(|A|=1)
$$

and

$$
\chi_{\tau}(A \cup B) \leqslant x_{\tau}(A \cup C)+x_{\tau}(B \cup C)
$$

hold for all $A, B, C \in \mathcal{F}\left(\Omega_{p, \tilde{r}}^{X}\right)$.

Let $|A|=1$. Then we have $A=\{\alpha\}$ for some $\alpha \in \Omega_{p, \tilde{r}}^{X}$. If $\tilde{x}=\left(x_{m}\right)_{m \in \mathbb{N}} \in \tilde{X}_{p, \tilde{r}}$ and $\pi(\tilde{x})=\alpha$, then (4.2) and property (i $\left.\mathrm{i}_{2}\right)$ of the ultrafilters imply

$$
x_{\tau}(A)=\mathfrak{U}-\lim \frac{\tau\left(\left\{x_{m}\right\}\right)}{r_{m}}=\mathfrak{U}-\lim 0=0 .
$$

Suppose now that $A$ has at least two distinct points $\alpha_{1}=\pi\left(\left(x_{m}^{1}\right)_{m \in \mathbb{N}}\right)$ and $\alpha_{2}=$ $\pi\left(\left(x_{m}^{2}\right)_{m \in \mathbb{N}}\right)$ where $\left(x_{m}^{1}\right)_{m \in \mathbb{N}},\left(x_{m}^{2}\right)_{m \in \mathbb{N}} \in \tilde{X}_{p, \tilde{r}}$. Then inequality (4.7) implies

$$
\frac{\tau\left(\operatorname{Im}\left(x_{m}^{1}, \ldots, x_{m}^{n}\right)\right)}{r_{m}} \geqslant \frac{1}{2} \frac{d\left(x_{m}^{1}, x_{m}^{2}\right)}{r_{m}}
$$

for every $m \in \mathbb{N}$. By the definition we have

$$
\lim _{m \rightarrow \infty} \frac{1}{2} \frac{d\left(x_{m}^{1}, x_{m}^{2}\right)}{r_{m}}=\frac{1}{2} \rho\left(\alpha_{1}, \alpha_{2}\right)>0 .
$$

Hence all limit points of the sequence with the common term

$$
\frac{\tau\left(\operatorname{Im}\left(x_{m}^{1}, \ldots, x_{m}^{n}\right)\right)}{r_{m}}
$$

are positive. Therefore, by Lemma 4.2 , we obtain the strict inequality $X_{\tau}(A)>0$. Equivalence (4.9) is proved. 
Similarly, considering the limit points of the sequence that defines the value

$$
\left(X_{\tau}(A \cup B)-X_{\tau}(A \cup C)-X_{\tau}(B \cup C)\right)
$$

and using (1.2) and $\left(\mathrm{i}_{3}\right)$ we obtain (4.10). Thus $\mathcal{X}_{\tau}$ is an extended by Balk metric on $\Omega_{p, \tilde{r}}^{X}$.

To complete the proof it remains to check that $X_{\tau}$ is compatible with $\rho$. Let $\alpha_{1}, \alpha_{2} \in \Omega_{p, \tilde{r}}^{X}$ and $\alpha_{1}=\pi\left(\left(x_{m}^{1}\right)_{m \in \mathbb{N}}\right), \alpha_{2}=\pi\left(\left(x_{m}^{2}\right)_{m \in \mathbb{N}}\right)$ where $\left(x_{m}^{1}\right)_{m \in \mathbb{N}},\left(x_{m}^{2}\right)_{m \in \mathbb{N}} \in$ $\tilde{X}_{p, \tilde{r}}$. Then from $(1.7),(1.8),(4.2)$ and the fact that $\tau$ is compatible with $d$ we find

$$
\begin{gathered}
x_{\tau}\left(\left\{\alpha_{1}, \alpha_{2}\right\}\right)=\mathfrak{U}-\lim \frac{\tau\left(\operatorname{Im}\left(x_{m}^{1}, x_{m}^{2}\right)\right)}{r_{m}} \\
=\mathfrak{U}-\lim \frac{d\left(x_{m}^{1}, x_{m}^{2}\right)}{r_{m}}=\lim _{m \rightarrow \infty} \frac{d\left(x_{m}^{1}, x_{m}^{2}\right)}{r_{m}}=\rho\left(\alpha_{1}, \alpha_{2}\right),
\end{gathered}
$$

which is what had to be proved.

It is rather easy to show if an extended by Balk metric $\tau: \mathcal{F}(X) \rightarrow \mathbb{R}$ is generated by a metric $d: X^{2} \rightarrow \mathbb{R}$, then for all pretangent spaces $\left(\Omega_{p, \tilde{r}}^{X}, \rho\right)$ and nontrivial ultrafilters $\mathfrak{U}$ the extended metrics $X_{\tau}$ are generated by $\rho$. On the other hand if the space $(X, d)$ is discrete, then every pretangent space $\Omega_{p, \tilde{r}}^{X}$ is single-point. Consequently $X_{\tau}$ is generated by the metric $\rho$ as the extended by Balk metric on the single-point space, irrespective of whether $\tau$ is generated by the metric $d$ or not.

To describe the class of extended metrics $\tau: X \times X \rightarrow \mathbb{R}$ for which $X_{\tau}$ is generated by $\rho$, we need some "infinitesimal" variant of Definition 2.13.

Let $(X, d)$ be a metric space, $p \in X$ and $\tau: \mathcal{F}(X) \rightarrow \mathbb{R}$ be an extended metric for which $\tau^{2}=d$, i.e. $\tau$ is compatible with $d$.

Definition 4.6. The extended metric $\tau$ is generated by the metric $d$ at the point $p$ if for every $n \in \mathbb{N}$ and every finite set of sequences $\left(x_{m}^{1}\right)_{m \in \mathbb{N}}, \ldots,\left(x_{m}^{n}\right)_{m \in \mathbb{N}}$ which converge to $p$ with $m \rightarrow \infty$, the relation

$$
\left|\tau\left(\operatorname{Im}\left(x_{m}^{1}, \ldots, x_{m}^{n}\right)\right)-\operatorname{diam}_{d}\left(\operatorname{Im}\left(x_{m}^{1}, \ldots, x_{m}^{n}\right)\right)\right|=o\left(\max \left\{d\left(x_{m}^{1}, p\right), \ldots, d\left(x_{m}^{n}, p\right)\right\}\right)
$$

holds, where $\operatorname{Im}\left(x_{m}^{1}, \ldots, x_{m}^{n}\right)$ is defined by (1.5).

Remark 4.7. Relation (4.11) means that

$$
\lim _{m \rightarrow \infty} \frac{\left|\tau\left(\operatorname{Im}\left(x_{m}^{1}, \ldots, x_{m}^{n}\right)\right)-\operatorname{diam}_{d}\left(\operatorname{Im}\left(x_{m}^{1}, \ldots, x_{m}^{n}\right)\right)\right|}{\max \left\{d\left(x_{m}^{1}, p\right), \ldots, d\left(x_{m}^{n}, p\right)\right\}}=0,
$$

with $\lim _{m \rightarrow \infty} \frac{\left|\tau\left(\operatorname{Im}\left(x_{m}^{1}, \ldots, x_{m}^{n}\right)\right)-\operatorname{diam}_{d}\left(\operatorname{Im}\left(x_{m}^{1}, \ldots, x_{m}^{n}\right)\right)\right|}{\max \left\{d\left(x_{m}^{1}, p\right), \ldots, d\left(x_{m}^{n}, p\right)\right\}}:=0$ for $x_{m}^{1}=\ldots=x_{m}^{n}=p$.

Theorem 4.8. Let $X \neq \varnothing, p \in X$ and let $\tau: \mathcal{F}(X) \rightarrow \mathbb{R}$ be an extended by Balk metric. Then the following statements are equivalent. 
(i) For every nontrivial ultrafilter $\mathfrak{U}$ on $\mathbb{N}$ and every space $\left(\Omega_{p, \tilde{r}}^{X}, \rho\right)$ which is pretangent to the metric space $\left(X, \tau^{2}\right)$ at the point $p$, the extended metric $X_{\tau}: \mathcal{F}\left(\Omega_{p, \tilde{r}}^{X}\right) \rightarrow \mathbb{R}$ is generated by $\rho$.

(ii) The extended metric $\tau$ is generated by the metric $\tau^{2}$ at the point $p$.

Proof. For convenience write $d:=\tau^{2}$.

First consider the case when $p$ is an isolated point of the space $(X, d)$. In this case the equality $x_{m}=p$ holds for every sequence $\left(x_{m}\right)_{m \in \mathbb{N}} \in \tilde{X}_{p}$ if $m \in \mathbb{N}$ is sufficiently large. Using Remark 4.7 we see that $\tau$ is generated by $d$ at the point $p$. For isolated $p$ any $\Omega_{p, \tilde{r}}^{X}$ is a single-point space. Property (ii) holds. To prove (i) observe, that for the single-point space there is the unique extended metric which is generated by the unique metric on such space.

Let us turn now to less trivial case when $p$ is a limit point of $X$. Suppose (ii) holds. Consider an arbitrary pretangent space $\left(\Omega_{p, \tilde{r}}^{X}, \rho\right)$, a nontrivial ultrafilter $\mathfrak{U}$ on $\mathbb{N}$ and the extended metric $X_{\tau}: \mathcal{F}\left(\Omega_{p, \tilde{r}}^{X}\right) \rightarrow \mathbb{R}$ which is defined by $\mathfrak{U}$ according to (4.2).

We must prove the equality

$$
x_{\tau}(A)=\operatorname{diam}_{\rho} A
$$

for arbitrary $A=\left\{\alpha_{1}, \ldots, \alpha_{n}\right\} \in \mathcal{F}\left(\Omega_{p, \tilde{r}}^{X}\right)$.

By Theorem 4.3, $x_{\tau}$ is compatible with $\rho$. Therefore (4.13) holds for $n \leqslant$ 2. So we can assume $n \geqslant 3$. Let $\tilde{X}_{p, \tilde{r}}$ be a maximal self-stable family in $\tilde{X}_{p}$ corresponding to $\Omega_{p, \tilde{r}}^{X}$ and let $\pi: \tilde{X}_{p, \tilde{r}} \rightarrow \Omega_{p, \tilde{r}}^{X}$ be the projection that maps the sequences $\left(x_{m}\right)_{m \in \mathbb{N}} \in \tilde{X}_{p, \tilde{r}}$ to their equivalence classes (see (1.8)). Relation (4.13) can be rewritten as

$$
\mathfrak{U}-\lim \frac{\tau\left(\operatorname{Im}\left(x_{m}^{1}, \ldots, x_{m}^{n}\right)\right)}{r_{m}}=\operatorname{diam}_{\rho} A,
$$

where $r_{m}$ and $x_{m}^{1}, \ldots, x_{m}^{n}$ are the $m$-th elements of the normalizing sequence $\tilde{r}=$ $\left(r_{m}\right)_{m \in \mathbb{N}}$ and, respectively, of the sequences $\left(x_{m}^{1}\right)_{m \in \mathbb{N}}, \ldots,\left(x_{m}^{n}\right)_{m \in \mathbb{N}} \in \tilde{X}_{p, \tilde{r}}$ for which $\pi\left(\left(x_{m}^{i}\right)\right)=\alpha_{i}, i=1, \ldots, n$. The following limit relations directly follow from the definition of the metric $\rho$ on $\Omega_{p, \tilde{r}}^{X}$,

$$
\begin{gathered}
\operatorname{diam}_{\rho} A=\lim _{m \rightarrow \infty} \frac{\operatorname{diam}_{d}\left(\operatorname{Im}\left(x_{m}^{1}, \ldots, x_{m}^{n}\right)\right)}{r_{m}}, \\
\max \left(\left\{\rho\left(\alpha, \alpha_{1}\right), \ldots, \rho\left(\alpha, \alpha_{n}\right)\right\}\right)=\lim _{m \rightarrow \infty} \frac{\max \left(\left\{d\left(p, x_{m}^{1}\right), \ldots, d\left(p, x_{m}^{n}\right)\right\}\right)}{r_{m}},
\end{gathered}
$$

where $\alpha=\pi(\tilde{p}), \tilde{p}=(p, p, p \ldots)$. Using properties $\left(\mathrm{i}_{2}\right),\left(\mathrm{i}_{3}\right)$ of the ultrafilters, equalities (1.8) and (4.2) and the first equality from (4.15), we can rewrite (4.14) in the form

$$
\mathfrak{U}-\lim \frac{\tau\left(\operatorname{Im}\left(x_{m}^{1}, \ldots, x_{m}^{n}\right)\right)-\operatorname{diam}_{d}\left(\operatorname{Im}\left(x_{m}^{1}, \ldots, x_{m}^{n}\right)\right)}{r_{m}}=0 .
$$


Since $n \geqslant 3$, then the strict inequality

$$
\max \left\{d\left(x_{m}^{1}, p\right), \ldots, d\left(x_{m}^{n}, p\right)\right\}>0
$$

holds for sufficient large $m$. Using this inequality, (4.12) and the second equality from (4.15) we find

$$
\begin{gathered}
\lim _{m \rightarrow \infty} \frac{\tau\left(\operatorname{Im}\left(x_{m}^{1}, \ldots, x_{m}^{n}\right)\right)-\operatorname{diam}_{d}\left(\operatorname{Im}\left(x_{m}^{1}, \ldots, x_{m}^{n}\right)\right)}{r_{m}}= \\
\lim _{m \rightarrow \infty}\left(\frac{\tau\left(\operatorname{Im}\left(x_{m}^{1}, \ldots, x_{m}^{n}\right)\right)-\operatorname{diam}_{d}\left(\operatorname{Im}\left(x_{m}^{1}, \ldots, x_{m}^{n}\right)\right)}{\max \left\{d\left(x_{m}^{1}, p\right), \ldots, d\left(x_{m}^{n}, p\right)\right\}} \frac{\max \left\{d\left(x_{m}^{1}, p\right), \ldots, d\left(x_{m}^{n}, p\right)\right\}}{r_{m}}\right) \\
=0 \cdot \max \left\{\rho\left(\alpha, \alpha_{1}\right), \ldots, \rho\left(\alpha, \alpha_{n}\right)\right\}=0 .
\end{gathered}
$$

Now property $\left(\mathrm{i}_{2}\right)$ of the ultrafilters implies (4.16). The implication (ii) $\Rightarrow$ (i) is proved.

To complete the proof it remains to establish the converse implication (i) $\Rightarrow$ (ii). Suppose that (i) is true but (ii) is false. Then there are an integer number $n \geqslant 3$ and sequences $\left(x_{m}^{i}\right)_{m \in \mathbb{N}} \in \tilde{X}_{p}, i=1, \ldots, n$ such that a limit point $b$ of the sequence $\left(y_{m}\right)_{m \in \mathbb{N}}$

$$
y_{m}=\frac{\tau\left(\operatorname{Im}\left(x_{m}^{1}, \ldots, x_{m}^{n}\right)\right)-\operatorname{diam}_{d}\left(\operatorname{Im}\left(x_{m}^{1}, \ldots, x_{m}^{n}\right)\right)}{\max \left\{d\left(x_{m}^{1}, p\right), \ldots, d\left(x_{m}^{n}, p\right)\right\}},
$$

is nonzero, $b \neq 0$. The sequence $\left(y_{m}\right)_{m \in \mathbb{N}}$ is bounded. Indeed, if $\operatorname{diam}_{d}\left(\operatorname{Im}\left(x_{m}^{1}, \ldots, x_{m}^{n}\right)\right)=d\left(x_{m}^{i_{1}}, x_{m}^{i_{2}}\right), 1 \leqslant i_{1}, i_{2} \leqslant n$, then

$$
0 \leqslant \frac{\operatorname{diam}_{d}\left(\operatorname{Im}\left(x_{m}^{1}, \ldots, x_{m}^{n}\right)\right)}{\max \left\{d\left(x_{m}^{1}, p\right), \ldots, d\left(x_{m}^{n}, p\right)\right\}} \leqslant \frac{d\left(x_{m}^{i_{1}}, p\right)+d\left(x_{m}^{i_{2}}, p\right)}{\max \left\{d\left(x_{m}^{1}, p\right), \ldots, d\left(x_{m}^{n}, p\right)\right\}} \leqslant 2 .
$$

Similarly, using (4.3) we find

$$
\tau\left(\operatorname{Im}\left(x_{m}^{1}, \ldots, x_{m}^{n}\right)\right) \leqslant \sum_{i=1}^{n-1} \tau\left(\operatorname{Im}\left(x_{m}^{i}, x_{m}^{i+1}\right)\right)=\sum_{i=1}^{n-1} d\left(x_{m}^{i}, x_{m}^{i+1}\right),
$$

that gives

$$
\begin{gathered}
0 \leqslant \frac{\tau\left(\operatorname{Im}\left(x_{m}^{1}, \ldots, x_{m}^{n}\right)\right)}{\max \left\{d\left(x_{m}^{1}, p\right), \ldots, d\left(x_{m}^{n}, p\right)\right\}} \leqslant \sum_{i=1}^{n-1} \frac{d\left(x_{m}^{i}, x_{m}^{i+1}\right)}{\max \left\{d\left(x_{m}^{1}, p\right), \ldots, d\left(x_{m}^{n}, p\right)\right\}} \\
\leqslant \sum_{i=1}^{n-1} \frac{d\left(x_{m}^{i}, p\right)+d\left(x_{m}^{i+1}, p\right)}{\max \left\{d\left(x_{m}^{1}, p\right), \ldots, d\left(x_{m}^{n}, p\right)\right\}} \leqslant 2(n-1) .
\end{gathered}
$$

Inequalities (4.17) and (4.18) imply the desirable boundedness. Passing from $\left(y_{m}\right)_{m \in \mathbb{N}}$ to a suitable subsequence of $\left(y_{m}\right)_{m \in \mathbb{N}}$ it can be assumed that

$$
\lim _{m \rightarrow \infty} y_{m}=b \quad \text { and } \quad b \notin\{0,+\infty,-\infty\}
$$


Moreover, using the conditions $\left(x_{m}^{i}\right)_{m \in \mathbb{N}} \in \tilde{X}_{p}, i=1, \ldots, n$ and passing to a subsequence again we can assume $\lim _{m \rightarrow \infty} \max \left\{d\left(x_{m}^{1}, p\right), \ldots, d\left(x_{m}^{n}, p\right)\right\}=0$ and $\max \left\{d\left(x_{m}^{1}, p\right), \ldots, d\left(x_{m}^{n}, p\right)\right\}>0$ for $m \in \mathbb{N}$.

Thus the sequence $\left(r_{m}\right)_{m \in \mathbb{N}}$ with $r_{m}=\max \left\{d\left(x_{m}^{1}, p\right), \ldots, d\left(x_{m}^{n}, p\right)\right\}$ can be selected as normalizing. Using the obvious inequalities

$$
\frac{d\left(x_{m}^{i}, x_{m}^{j}\right)}{r_{m}} \leqslant 2 \quad \text { and } \quad \frac{d\left(x_{m}^{i}, p\right)}{r_{m}} \leqslant 1
$$

and passing to a subsequence again we can assume that the sequences $\tilde{p}, \tilde{x}_{1}=$ $\left(x_{m}^{1}\right)_{m \in \mathbb{N}}, \ldots, \tilde{x}_{n}=\left(x_{m}^{n}\right)_{m \in \mathbb{N}}$ are mutually stable. Let $\tilde{X}_{p, \tilde{r}}$ be a maximal selfstable family for which $\tilde{x}_{i} \in \tilde{X}_{p, \tilde{r}}, i=1, \ldots, n$ and $\Omega_{p, \tilde{r}}^{X}$ be the corresponding pretangent space. Let $\mathfrak{U}$ be a nontrivial ultrafilter on $\mathbb{N}$. Denote by $\alpha_{i}$ the image of subsequence $\tilde{x}_{i}=\left(x_{m}^{i}\right)_{m \in \mathbb{N}}$ under the projection of $\tilde{X}_{p, \tilde{r}}$ on $\Omega_{p, \tilde{r}}^{X}, \alpha_{i}=\pi\left(\tilde{x}_{i}\right)$. Now using properties $\left(i_{2}\right)-\left(i_{3}\right)$ and equality (4.19) we obtain

$$
\begin{gathered}
b=\mathfrak{U}-\lim \frac{\tau\left(\operatorname{Im}\left(x_{m}^{1}, \ldots, x_{m}^{n}\right)\right)-\operatorname{diam}_{d}\left(\operatorname{Im}\left(x_{m}^{1}, \ldots, x_{m}^{n}\right)\right)}{r_{m}} \\
=\left(\mathfrak{U}-\lim \frac{\tau\left(\operatorname{Im}\left(x_{m}^{1}, \ldots, x_{m}^{n}\right)\right)}{r_{m}}\right)-\left(\mathfrak{U}-\lim \frac{\operatorname{diam}_{d}\left(\operatorname{Im}\left(x_{m}^{1}, \ldots, x_{m}^{n}\right)\right)}{r_{m}}\right) \\
=X_{\tau}\left(\left\{\alpha_{1}, \ldots, \alpha_{n}\right\}\right)-\operatorname{diam}_{\rho}\left(\left\{\alpha_{1}, \ldots, \alpha_{n}\right\}\right) .
\end{gathered}
$$

Since $b \neq 0$ it implies the relation

$$
X_{\tau}\left(\left\{\alpha_{1}, \ldots, \alpha_{n}\right\}\right) \neq \operatorname{diam}_{\rho}\left(\left\{\alpha_{1}, \ldots, \alpha_{n}\right\}\right),
$$

contrary to (i).

The implication (i) $\Rightarrow$ (ii) follows.

Theorem 4.8 and some known results about pretangent spaces allow, in some cases, to get the relatively simple answer to the question about infinitesimal structure of extended metrics $\tau: \mathcal{F}(X) \rightarrow \mathbb{R}$ for which the corresponding extended metrics $X_{\tau}$ on pretangent spaces are generated by metrics with some special properties.

Recall that a metric space $(X, d)$ is called ultrametric if the inequality

$$
d(x, y) \leqslant d(x, z) \vee d(z, y)
$$

holds for all $x, y, z \in X$. Here and in the sequel we set $p \vee q=\max \{p, q\}$ and $p \wedge q=\min \{p, q\}$ for all $p, q \in \mathbb{R}$.

Let $(X, d)$ be a metric spaces with a marked point $p$. Let us define a function $F_{d}: X^{3} \rightarrow \mathbb{R}$ as

$$
F_{d}(x, y):=\left\{\begin{array}{lll}
\frac{d(x, y)(d(x, p) \wedge d(y, p))}{(d(x, p) \vee d(y, p))^{2}} & \text { if } & (x, y) \neq(p, p) \\
0 & \text { if } & (x, y)=(p, p)
\end{array}\right.
$$


and a function $\Phi_{d}: X^{3} \rightarrow \mathbb{R}$ as $\Phi_{d}(x, y, z):=F_{d}(x, y) \vee F_{d}(x, z) \vee F_{d}(y, z)$ for every $(x, y, z) \in X^{3}$. For convenience we introduce the notations: $d_{1}(x, y, z)$ is length of greatest side of the triangle with the sides $d(x, y), d(x, z)$ and $d(y, z)$ and $d_{2}(x, y, z)$ is length of greatest of the two remained sides of this triangle.

Lemma 4.9.([11]) Let $(X, d)$ be a metric space with a marked point $p$. All pretangent spaces $\Omega_{p, \tilde{r}}^{X}$ are ultrametric if and only if

$$
\lim _{x, y, z \rightarrow p} \Phi_{d}(x, y, z)\left(\frac{d_{1}(x, y, z)}{d_{2}(x, y, z)}-1\right)=0,
$$

where $\frac{d_{1}(x, y, z)}{d_{2}(x, y, z)}:=1$ for $d_{2}(x, y, z)=0$.

Using Theorem 4.8 and Lemma 4.9 we get

Corollary 4.10. Let $X \neq \varnothing, p \in X$ and let $\tau: \mathcal{F}(X) \rightarrow \mathbb{R}$ be an extended by Balk metric. The following statements are equivalent.

(i) All extended metrics $\mathcal{X}_{\tau}: \mathcal{F}\left(\Omega_{p, \tilde{r}}^{X}\right) \rightarrow \mathbb{R}$ are generated by ultrametrics.

(ii) The extended metric $\tau: \mathcal{F}(X) \rightarrow \mathbb{R}$ is generated by the metric $\tau^{2}$ at the point $p$ and the equality

$$
\lim _{x, y, z \rightarrow p} \Phi_{\tau^{2}}(x, y, z)\left(\frac{\tau_{1}^{2}(x, y, z)}{\tau_{2}^{2}(x, y, z)}-1\right)=0,
$$

holds with $\frac{\tau_{1}^{2}(x, y, z)}{\tau_{2}^{2}(x, y, z)}:=1$ for $x=y=z=p$.

Remark 4.11. An extended metric $X_{\tau}: \mathcal{F}\left(\Omega_{p, \tilde{r}}^{X}\right) \rightarrow \mathbb{R}$ is generated by an ultrametric if and only if the inequality

$$
\chi_{\tau}(A \cup B) \leqslant \mathcal{X}_{\tau}(A \cup C) \vee \mathcal{X}_{\tau}(B \cup C)
$$

holds for all $A, B, C \in \mathcal{F}\left(\Omega_{p, \tilde{r}}^{X}\right)$. (See Theorem 2.1 in [8]).

Acknowledgements. The research of the first author was supported by a grant received from TUBITAK within 2221-Fellowship Programme for Visiting Scientists and Scientists on Sabbatical Leave and by project 15-1 bb \19, "Metric Spaces, Harmonic Analysis of Functions and Operators and Singular and Non-classic Problems for Differential Equations", Donetsk National University (Vinnitsa, Ukraine).

\section{References}

[1] F. Abdullayev, O. Dovgoshey, M. Küçükaslan, Metric spaces with unique pretangent spaces. Conditions of the uniqueness, Ann. Acad. Sci. Fenn. Math., 36(2011), 353-392. 
[2] P. I. Balk, Conseptual evaluation of $\varepsilon$-equivalency in non-linear inverse gravity problems, Geophysical Journal, 31(6)(2009), 55-61.

[3] P. I. Balk, On an extension of the concept of metric (Russian), Dokl. Math., 79(3)(2009), 394-396.

[4] V. V. Bilet, Geodesic spaces tangent to metric spaces, Ukr. Math. J., 64(9)(2013), 1448-1456.

[5] V. Bilet, O. Dovgoshey, Isometric embeddings of pretangent spaces in $E^{n}$, Bull. Belg. Math. Soc. - Simon Stevin, 20(2013), 91-110.

[6] B. C. Dhage, Generalized metric space and mapping with fixed point, Bull. Calcutta Math. Soc., 84(4)(1992), 329-336.

[7] Dmitrii V. Dordovski, Metric tangent spaces to Euclidean spaces, J. Math. Sci. (N. Y.), 179(2)(2011), 229-244.

[8] D. Dordovskyi, O. Dovgoshey, E. Petrov, Diameter and Diametrical Pairs of Points in Ultrametric Spaces, p-Adic Numbers, Ultrametric Anal. App., 3(4)(2011), 253-262.

[9] O. Dovgoshey, Tangent spaces to metric spaces and to their subspaces, Ukr. Math. Bull., 5(4)(2008), 470-487.

[10] O. Dovgoshey, F. Abdullayev, M. Küçükaslan, Compactness and boundedness of tangent spaces to metric spaces, Beiträge Algebra Geom., 51(2)(2010), 547-576.

[11] A. A. Dovgoshey, D. V. Dordovskyi, An ultrametricity condition for pretangent spaces, Math. Notes, 92(7)(2012), 43-50.

[12] O. Dovgoshey, O. Martio, Tangent spaces to metric spaces, Reports in Math., Helsinki Univ., 480(2008), 1-20.

[13] O. Dovgoshey, O. Martio, Tangent spaces to general metric spaces, Rev. Roum. Math. Pures. Appl., 56(2)(2011), 137-155.

[14] S. Gahler, 2-metrische Räume und ihre topologische Structur, Mathematische Nachrichten, 26(1963), 115-148.

[15] S. Gahler, Zur geometric 2-metriche raume, Revue Roum. Math. Pures App., 40(1966), 664-669.

[16] J. L. Kelly, General Topology, D. Van Nostrand Company, 1965.

[17] P. Komjáth, V. Totik, Problems and Theorems in Classical Set Theory, Springer, New York, 2006.

[18] Z. Mustafa, A new structure for generalized metric spaces - with applications to fixed point theory, Ph. D. thesis, The University of Newcastle, Callaghan, Australia, 2005.

[19] Z. Mustafa, O. Hamed, F. Awawdeh, Some fixed point theorem for mapping on complete G-metric spaces, Fixed Point Theory App., 2008(2008), Article ID 189870, 12p. 1.

[20] Z. Mustafa, B. Sims, A new approach to generalized metric spaces, J. Nonlinear Convex Anal., 7(2)(2006), 289-297. 